Editorial

\title{
The liquid biopsy for tracking early molecular changes in malignant tumors
}

Volume 7 Issue 3 - 2017

\section{Introduction}

A biopsy, which consists in taking from a tumor tissue samples and analyzing them, is one way to track whether the cancer is responding to treatment or growing resistant. But that's a highly invasive procedure that can't reasonably be done so frequently. For most tumors, a tissue biopsy is quite challenging, in that it's costly, painful, and potentially risky for the patient. To date, the liquid biopsy, a blood test that measures the serum level of circulating tumor (ct) DNA, has generated a lot of excitement. Research shows how it can provide a noninvasive, ongoing picture of a patient's cancer especially when the cancer is in a difficult-to-reach location such as the brain, offering valuable insight into how best to fight it. One area the two types of biopsies do not agree, involves mutations indicating resistance to therapies. Those were picked up in the blood tests but not in the tissue samples; perhaps because those changes occurred after the standard biopsies were completed and treatment was initiated.

\section{Liquid cancer test, an alternative}

DNA fragments from tumors can be found in tiny amounts in the bloodstream of patients with cancer. Such tests are designed to pick up these small pieces in the bloodstream; the analysis of information procured, can then be used to treat and monitor the disease. The only liquid biopsy currently approved by the US Food and Drug Administration (FDA) for clinical use is a prognostic survival tool with no potential to guide treatment decisions. Work from 2 different groups, Dr. Diaz and colleagues and Sandra Misale and colleagues, shows how liquid biopsies are being used in the lab to identify and monitor tumors at a very early stage, even to find out signs of early treatment resistance.

"Mutant DNA fragments are found at relatively high concentrations in the circulation of most patients with metastatic cancer and at lower but detectable concentrations in a substantial fraction of patients with localized cancers," Dr. Diaz and colleagues declare. This being particularly applicable to a certain number of cancers such as breast, colon, pancreas, and gastroesophageal tumors, in which "detectable levels of ctDNA were present in $49 \%$ to $78 \%$ of patients with localized tumors and $86 \%$ to $100 \%$ of patients with metastatic tumors."

Bettegowda et al., ${ }^{1}$ measured levels of ctDNA with tumour-specific DNA mutations or structural changes using a sophisticated method of digital polymerase chain reaction-based sequencing techniques and rearrangement analyses, in large cohorts of matched human plasma and tumour tissue samples. ctDNA was detected in " $82 \%$ of patients with solid tumours outside the brain, including more than $75 \%$ of patients with advanced ovarian, colorectal, bladder, gastroesophageal, pancreatic, breast, hepatocellular, and head and neck cancers, as well as melanomas. Less than $50 \%$ of patients with medulloblastomas or metastatic cancers of the kidney, prostate or thyroid, and less than $10 \%$ of patients with gliomas, had detectable ctDNA". Patients with localized cancer of all types evaluated, "only 55\% had detectable ctDNA, but the proportion of patients with detectable ctDNA increased with tumour stage". Of note, CTCs and ctDNA seemed to be two biomarkers of distinct nature.

\author{
Hajj Adel Anis \\ Cedars-Jebel Ali International Hospital, UAE
}

Correspondence: Haji Adel Anis, Medical Oncologist at Cedars - Jebel Ali International Hospital, 9370 Rue Lajeunesse, Montreal, UAE, Tel 438-992-55I6, Email ahajj@dr.com

Received: January 31, 2017 | Published: February 17, 2017

In addition, liquid biopsies offer indexes to the reasons for treatment resistance. This is how researchers showed, in colorectal cancer patients, that early resistance to treatment with epidermal growth-factor receptor (EGFR) inhibitors could be identified by the presence of certain mutations in the blood. They note that liquid biopsies are "more likely to capture the overall genetic complexity of tumors in patients with advanced disease. ${ }^{2-5}$

\section{Conclusion}

ctDNA investigations in cancer patients, through liquid biopsies, are increasingly being performed, poiting out clearly the advantages and the different potential applications of this approach. Serial analysis of ctDNA can provide a dynamic picture of molecular disease changes in real time, which is a very big step beyond where CTCs are today. ctDNA could potentially be used to identify genomic targets for treatment. In the future, instead of extensive imaging and invasive tissue biopsies, liquid biopsies could be used to guide cancer treatment decisions and perhaps even screen for tumors that are not yet visible on imaging.

\section{Acknowledgments}

None.

\section{Conflicts of interest}

Author declares there are no conflicts of interest.

\section{References}

1. Bettegowda C, Sausen M, Leary RJ, et al. Detection of Circulating Tumor DNA in Early- and Late-Stage Human Malignancies. Sci Transl Med. 2014;6:224ra24.

2. Misale S, Sabrina Arena S, Simona Lamba S, et al. Blockade of EGFR and MEK Intercepts Heterogeneous Mechanisms of Acquired Resistance to Anti-EGFR Therapies in Colorectal Cancer. Sci Transl Med. 2014;6:224ra26. 
3. Niki Karachaliou, Clara Mayo-de-las-Casas, Miguel Angel Molina-Vila et al. Real-time liquid biopsies become a reality in cancer treatment. Ann Transl Med. 2015;3(3):36.

4. Misale S, Arena S, Lamba S, et al. Blockade of EGFR and MEK intercepts heterogeneous mechanisms of acquired resistance to anti-EGFR therapies in colorectal cancer. Sci Transl Med. 2014;6(224):224ra-26.
5. Ilie M, Hofman V, Long-Mira E, et al. Sentinel circulating tumor cells allow early diagnosis of lung cancer in patients with chronic obstructive pulmonary disease. PLoS One. 2014;9(10):e111597. 